\title{
Topological Data Analysis Gives Two Folding Paths in HP35(nle-nle), Double Mutant of Villin Headpiece Subdomain
}

Takashi Ichinomiya ( $\nabla$ tk1miya@gifu-u.ac.jp )

Gifu University School of Medicine

\section{Research Article}

Keywords: topological data analysis (TDA), matrix factorization, HP35(nle-nle),

Posted Date: December 3rd, 2021

DOl: https://doi.org/10.21203/rs.3.rs-514724/v1

License: (c) (i) This work is licensed under a Creative Commons Attribution 4.0 International License.

Read Full License

Version of Record: A version of this preprint was published at Scientific Reports on February 17th, 2022. See the published version at https://doi.org/10.1038/s41598-022-06682-x. 


\title{
Topological data analysis gives two folding paths in HP35(nle-nle), double mutant of villin headpiece subdomain
}

\author{
Takashi Ichinomiya ${ }^{1,2, *}$ \\ ${ }^{1}$ Department of Systems Biology, Gifu University School of Medicine, Yanagido 1-1, Gifu, 501-1194, Japan \\ ${ }^{2}$ The United Graduate School of Drug Discovery and Medical Information Sciences of Gifu University, Yanagido 1-1, \\ Gifu, 501-1194, Japan \\ *tk1miya@gifu-u.ac.jp
}

\begin{abstract}
The folding dynamics of proteins is a primary area of interest in protein science. We carried out topological data analysis (TDA) of the folding process of HP35(nle-nle), double-mutant of villin headpiece subdomain. Using persistent homology and non-negative matrix factorization, we reduced the dimension of protein structure into two, and investigate the flow in the reduced space. We found this protein has two folding paths, distinguished by the pairings of inter-helix residues. Our analysis showed the excellent performance of TDA in capturing the formation of tertiary structure.
\end{abstract}

\section{Introduction}

The folding mechanism of protein is a primary area of interest in protein science. Understanding the process of protein folding is essential for recognizing the role of proteins in living cells and developing new therapies for protein-misfolding diseases such as Creutzfeldt-Jakob disease or Altzheimer's disease ${ }^{1}$. Recent development of computer technology have enabled large-scale molecular-dynamics(MD) simulations for this purpose. However, it remains challenging to obtain essential information on the folding process from data obtained using MD.

MD simulation provides extensive data, which must be condensed to obtain essential information. Previous studies have attempted to identify the essential bonding that characterizes protein folding structure by trial and error. Data-scientific methods, such as principal component analysis (PCA) or k-means clustering were also used to study the folding structures. However, the nonlinearity and nonlocality of the folding process make it difficult to apply such methods. In protein folding, a small change in the local bending angle causes a large change in the position of all residues; and linear analysis, such as PCA or k-means, often provide insufficient information. There are several non-linear methods available, such as kernel PCA, isomap, or t-distrubuted stochastic neighbor embedding, but these require large computational costs and are not applicable when the number of samples exceeds $O\left(10^{4}\right)$.

Recently, topological data analysis(TDA) has attracted considerable attention in a variety of fields ${ }^{2}$. In TDA, the structure of the data is characterized using topological features, such as the number of loops or connected components. TDA has several advantages compared to other data analysis method for protein folding; first, it can capture the nonlocal characteristics in the data. In the standard analysis of protein folding, Cartesian coordinates or the dihedral angle of residues are used to characterize the structure, but these variables are defined by one, two or three residues. TDA captures the structure such as loops or cavities, which are defined as the set of many atoms or residues, are suitable for capturing nonlocal structure. Second, TDA has advantage in capturing "unfolded states". Usually, the unfolded state of protein has a large degree of freedom in motion because the chain of residues can bend almost freely. This large degree of freedom often makes standard analysis method such as PCA or k-means clustering difficult because they are strongly affected by the large fluctuation in this state. However, in the unfolded state, there are no or few stable loops, and the unfolded state can be labeled as a state without a loop in the TDA. Third, TDA provides intuitive insights because the "loop" or "cavity" can be intuitively recognized; thus, TDA gives comprehensible information on the structure.

In the last decade, several studies have attempted to apply TDA for the analysis of biomolecules, such as RNA or proteins. For example, Yao et al. analyzed the folding path of RNA using Mapper, a popular TDA method ${ }^{3}$. Xia and Wei proposed "topological fingerprints" to classify protein structures ${ }^{4,5}$. They also attempted to apply the combination of deep learning and TDA to classify the protein structure ${ }^{6}$. In our previous study, we proposed a feature construction based on persistent homology $(\mathrm{PH})$, the most widely used TDA ${ }^{7}$. We applied this method to the MD simulation of chignolin and identified the 


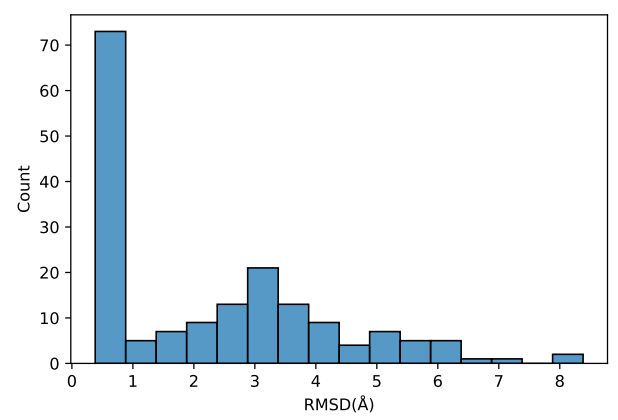

Figure 1. Distribution of minimum root mean square distance between trajectories and native state.

stable, meta-stable, and transition states.

In this paper, we applied our method to the dynamics of HP35(nle-nle), a double mutant of the villin headpiece. HP35 is a small protein consisting of 35 residue; it has three $\alpha$-helices, and is one of the first proteins whose folding processes are reproduced by MD simulation. However, the folding process of this protein is not completely understood. After the success of MD simulation by Beauchmap et al. ${ }^{8}$, several groups have been investigated the folding process of proteins in this group ${ }^{9,10}$. Although several experimental studies have suggested the existence of multiple folding paths, the theoretical results are controversial. Harada et al. present that there are two folding paths, distinguished by the order of the formation of $\alpha$-helices ${ }^{9}$. Wang et al. claimed to have identified a third path ${ }^{10}$. In these preceding studies, the paths are classified by the order of formation of $\alpha$-helices. However, it is not clear whether this order results in a different tertiary structure formation process. In this study, we analyzed the MD data of HP35(nle-nle) obtained by Beauchampl et al. using TDA. The result showed that there are two folding paths, characterized by the pairings between V9 and K32, F10 nad L28, F17 and L20, L20 and Q25, and F17 and Q25. These parings are inter-helix coupling and suggest the existence of multiple paths that have different tertiary structure.

\section{Methods}

\section{Data preprocessing}

The dataset provided by Beauchmap and Pande downloaded from Stanford University (https: / /exhibits. stanford. edu/data/catalog/bd829sf1034) was used. This dataset has 175 trajectories, and each trajectory is named as "RUN"+integer, such as RUN897 or RUN45767. Every trajectory has snapshots of atoms every 250 ps. The length of trajectories are not the same, and 1,352,345 snapshots were obtained in total. Before TDA, these trajectories were investigated and it was found that some trajectories failed to fold correctly. To remove unfolded trajectories, the root mean square distance(RMSD) between $\mathrm{C}^{\alpha}$ atoms in native state and those in every snapshots was calculated. The distribution of the minimal RMSD for each trajectory is shown in Fig.1. This figure shows that many trajectories were not correctly folded. Therefore, the trajectories whose minimum RMSD from native state is smaller than 1.0 Åwas selected. Moreover, all atoms had the same Cartesian coordinates in several snapshots; these snapshots were removed and 74 trajectories with 575,968 snapshots were obtained. These data sere used in the subsequent TDA.

\section{Persistent homology analysis}

To analyse the structure of protein, a PH analysis of the point cloud of residues was performed. Although PH can be formulated in pure mathematical framework ${ }^{11}$, here, $\mathrm{PH}$ is explained based on $\alpha$-filtration with degree 1 , used in the analysis.

First, the positions of $\mathrm{C}_{\alpha}$ atoms in protein were taken as those of residues. $\mathrm{In} \mathrm{PH}$, residues were approximated as spheres with radius $r$ and the change in topology when $r$ varies from 0 to $\infty$ was investigated. If $r$ was small, all residues were disconnected, as shown in Fig.2(a). By increasing $r$, the residues began to merge and a "cycle" surrounding the empty space will be formed, as indicated by the red triangle in Fig.2(b). However, a further increase in $r$ will destroy the cycle, as indicated by the red dashed triangle in Fig.2(c). This example demonstrated that every cycle had a "birth" and "death" radius. From one configuration of $\mathrm{C}^{\alpha}$ atoms, many cycles were obtained, and each was characterized by its birth and deaths. In the original PH, the set of births and deaths was used to characterize the topology of the data. In this approach, the "volume optimal cycle" was also used, depicted by the red polygon in Fig.2. The volume optimal cycle was defined as the "minimal" cycle that surrounds the empty space ${ }^{12}$. For example, in Fig.2(c), both red and yellow polygons surround the empty space. However, the red polygon is the sum of two triangle, while the yellow polygon is sum of three. Therefore red polygon is "smaller" than yellow one. In 


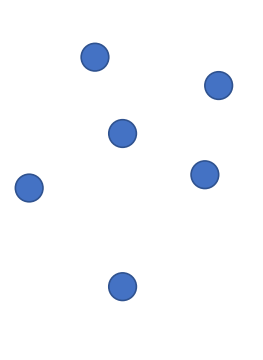

(a)

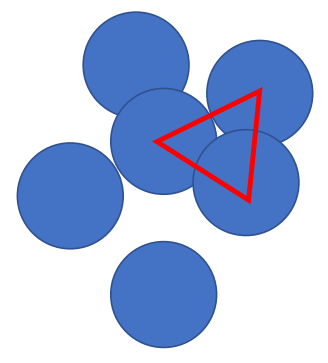

(b)

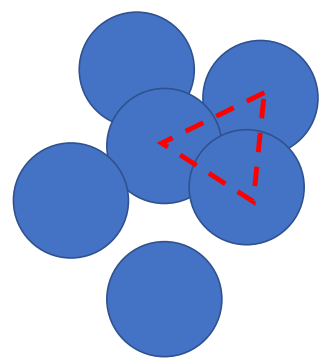

(c)

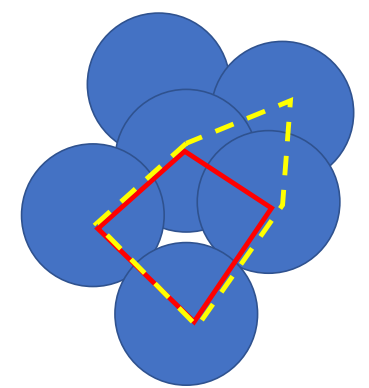

(d)

Figure 2. Schematic explanation of PH. (a): When the radius of balls $r$ was small, all balls were disconnected. (b): When $r$ increased, a cycle that surrounds the empty space is created, as indicated by the red triangle. (c): A further increase in $r$ destroyed the cycle. (d): Several polygons may surround same the empty space, as shown by the yellow and the red polygons. The volume optimal cycle was defined as the minimal cycle that surrounded the empty space. In this case, the red and yellow polygons consisted of two and three triangles, respectively, and the red polygon is the optimal one.

this case, red polygon represents the volume optimal cycle.

In this study, several assumptions regarding the $\mathrm{PH}$ of proteins were made. First, $\mathrm{PH}$ with degree 1, which provides information on the "loop". PH with different degrees provides other topological information, such as the number of connected components or cavities. However, loop information is especially useful for characterizing the structure of proteins ${ }^{4,7}$. For example, the formation of $\alpha$-helices or $\beta$-sheets can be characterized by the formation of a loop, while information on connected components or cavities does not provide insight into these structure. Therefore, information of PH with degree 1 was used. Second, each loop was characterized by birth and death in PH, and "lifetime," the difference between death and birth, was also considered as important variable in $\mathrm{PH}$, because it is related to the robustness against noise ${ }^{13}$. The cycle that emerged due to the small perturbation of atoms may have had a large birth or death values, but always had a small lifetime. Therefore, the lifetime was used for the vectorization explained in the next subsection. Third, although most of the application of PH mainly use births, deaths, and lifetimes to characterize the structure, the volume optimal cycle was also used, because births, deaths and lifeimes were insufficient to characterize the structure of HP35(nle-nle). One of the weakness of standard PH is that it cannot distinguish between similar structures. For example, the native state of HP35(nle-nle) has three $\alpha$-helices. Unfortunately, the cycles that emerged from these helices had similar births and deaths. Therefore, births and deaths provided insufficient information to distinguish the process of $\alpha$-helix formation. The volume optimal cycle provided the shape and location of loops, and helped to distinguish the order of $\alpha$-helix formation. The calculation of births, deaths, and volume optimal cycles were carried out by HomCloud 2.8.1 $1^{14}$.

\section{Vectorization of volume optimal cycles by "bag of simplices"}

For machine learning, information obtained by PH must be vectorized. Here, "bag of simplices", inspired by "bag of words" that is frequently used in natural language processing, was used ${ }^{7}$. In this approach, the set of volume optimal cycles was regarded as a "text" that describes the structure of a protein. Suppose that the protein structure had volume optimal cycles $C_{1}, C_{2}, \cdots C_{M}$. Every volume optimal cycle could be represented as a set of pairs of residues, $C_{k}=\left\{\left(i_{k_{1}}, j_{k_{1}}\right),\left(i_{k_{2}}, j_{k_{2}}\right), \cdots,\left(i_{k_{l}}, j_{k_{l}}\right)\right\}$. $s_{i, j}$ was defined as the "weight" of edge between $i$ and $j$, as

$$
s_{i j}=\sum_{C_{k} \ni(i, j)}\left(d_{k}-b_{k}\right),
$$

where $d_{k}$ and $b_{k}$ are the death and the birth of cycle $C_{k}$, respectively. Using this method, The volume optimal cycles were converted into $\frac{R(R-1)}{2}$-dimensional vectors, where $R$ denoted the number of residues. This procedure is shown in Fig.3. 

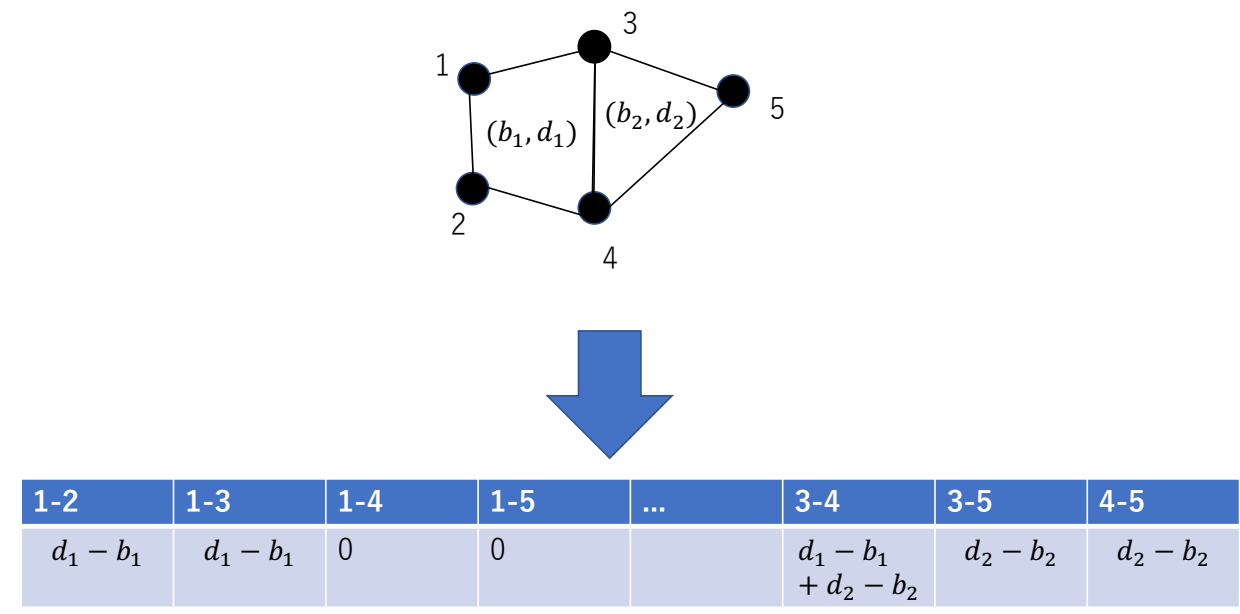

Figure 3. Definition of "bag of simplices".

\section{Dimension reduction by non-negative matrix factorization}

In the case of HP35(nle-nle), the feature vector obtained as a bag of simplices had $35 \times 34 / 2=595$ dimension. The dimension was so large that dimension reduction was needed to obtain comprehensible information. For this purpose, nonnegative matrix factorization(NMF) was used ${ }^{15}$. Suppose that there were $M$ samples whose $N$-dimensional feature vector was given by a nonnegative vector $v_{i}=\left(v_{i 1}, v_{i 2}, \cdots, v_{i N}\right)^{T}$, where $i$ denotes the index of the sample. In NMF, the nonnegative $N \times L$ matrix $W$ and $L \times M$ matrix $H$ that minimize $\|V-W H\|$, where $V=\left(v_{1}, v_{2}, \cdots, v_{M}\right)$, and $\|\cdots\|$ represents Frobenius norm, were calculated. Using NMF, the approximation $v_{i} \sim \sum_{j=1}^{L} w_{j} h_{j i}$ were obtained, where $w_{j}$ represent $j$-th column of matrix $W$.

Compared with other dimension-reduction techniques, such as PCA, manifold embedding, or deep learning, NMF has several advantages. First, it is easy to interpret the results of the dimension reduction. In PCA, the feature vectors were approximated by a linear combination of several vectors. This was similar to NMF, but negative components and coefficients in PCA often made it difficult to interpret the results. When attempting to approximate the feature vector $v$ using PCA, some components may become negative. If $v$ must be a nonnegative vector by definition, careful treatment is required to interpret the result. All coefficients and bases were nonnegative in NMF, and a negative component never appeared in the approximated feature vectors. Second, as shown by Lee and Seung, NMF often captures "local" structure, while PCA captures global structure $^{15}$. This property, which has not been proven rigorously, makes it easy to interpret the results. Third, the computational cost of NMF is low. NMF can be carried out at a lower computational cost than nonlinear analysis, such as manifold learning or deep learning.

Of course, NMF also has several disadvantages. First, NMF decomposition is not unique. If both $A$ and $A^{-1}$ are $L \times L$ non negative matrices, then $W^{\prime}=W A$ and $H^{\prime}=A^{-1} H$ are also non-negative, and another NMF is obtained. In particular, the scale of $H$ and $W$ was changed if $A$ was diagonal matrix, $A=\operatorname{diag}\left(\lambda_{1}, \lambda_{2}, \cdots, \lambda_{L}\right)$. NMF analysis was carried out several times and the consistency was checked in this study, and the result was rescaled to satisfy $\left|w_{i}\right|=1$ for $i=1,2, \cdots, L$. Therefore the unit of NMF scores presented in next section is $\AA$. Another problem in NMF is that there is not golden rule for determining the dimension of reduced space $L$. Here,the analysis for $L=4,5$ and 6 was attempted several times, and it was verified that the results of these analysis were qualitatively consistent. In this paper, NMF was performed using scikit-learn $0.24 .1^{16}$.

\section{Results}

\section{Density of states in reduced space}

Figure 4 shows the density of states in reduced space obtained by NMF for $L=5$. First, all histograms in the diagonal of Fig.4 had large strong peaks at 0 . This strong peak was also found in the case of chignolin, and was related to the unfolded state ${ }^{7}$. In the unfolded state, the number of cycles was small, and these cycles had a short lifetime. Therefore, almost all components 
of the "bag of simplices" vector became zero in the unfolded state, which resulted in a strong peak at 0 in the histogram. Second, the distribution of the scores of NMF1, NMF3, and NMF4 had two other peaks. The density of the states represented in the upper right triangle of Fig. 4 shows that there are several high density peaks in the space constructed from these three components, while the distribution of NMF2 and 5 did not show a clear structure. This observation was not modified when we changed the embedding dimension $L$ to 4 or 6 ; and we only considered on the reduced space spanned by NMF1, NMF3 and NMF4. Here, we note that the density obtained by this analysis was not directly related to the free energy. In the dataset, the simulation began from the unfolded state and stopped before reaching thermal equilibrium. Therefore, the densities shown in Fig.4 do not reflect the free energy, though the high-density state may be related to the meta-stable structure.

To identify the meaning of each component, the basis that corresponded to each component is shown in Fig.5. In this figure, the value of each component is visualized by color. For example, in Fig.5(a), the block at V9 and K32 is dark; therefore, the basis of NMF1 has a large value at V9-K32. Therefore, if a sample had a large NMF1 score, there were large loops that included the link between V9-K32. In the case of PCA, this conclusion should be reached carefully, because there may be a neglected basis that has a large negative value at V9-K32. In the case of NMF, every basis provides only a positive contribution, and we do not need to consider such a case.

From Fig.5, we first found that the vectors except NMF2 showed similar pairing between 3-residue-aparted residues, such as D3-F6 or Q25-L28. These pairs implied the formation of an $\alpha$-helix. In the native structure, as shown in Fig.6, these residues were close together along $\alpha$-helices. However, NMF2 did not show these pairs. Therefore, if an $\alpha$-helix is formed, the scores of NMF1, 3, 4 and 5 increased, while those of NMF2 increased when the loop without the $\alpha$-helix is formed. Second, the components of NMF1, 3 and 4 had pairs that characterized each state. For NMF1, the component at V9-K32 exceeded 0.3. Therefore, if there was a loop that involved these two residues, NMF1 score increased. Similarly, for NMF3, F10-L28 and F17-Q25 exceeded 0.3, and the NMF3 score increased when there were loops that included these edges. NMF4 score increased when there were loops with F17-L20 or L20-Q25. This suggestion is also supported by the correlation between NMF scores and weight of pairs in the bag of simplices, as shown in Table 1. The correlation between NMF1 score and weight of V9-K32 was 0.926, which suggests a strong correlation between these two variables. Similarly, NMF3, F10-L28, F17-Q25, NMF4, F17-L20, and L20-Q25 showed strong correlations, which supports our suggestion. To recognize the structure associated with these characteristic pairs, we showed these pairs in the native state in Fig.6. Overall, these pairs appeared to represent the formation of a tertiary structure. As shown in Fig.6, V9-K32 and F10-L28 pairs represented the contact between two $\alpha$-helices at the terminal subdomains. Pairs F17-L20, F17-Q25, and L20-Q25 represented the bending between second and C-terminus helix. NMF5 has no components that characterize its structure. Therefore, the large NMF5 implied that there is no clear tertiry structure, although $\alpha$-helices may exist.

\begin{tabular}{|c|c|c|c|c|c|}
\hline & V9-K32 & F10-L28 & F17-Q25 & F17-L20 & L20-Q25 \\
\hline \hline NMF1 & 0.926 & -0.420 & 0.350 & -0.067 & 0.010 \\
\hline NMF3 & -0.366 & 0.668 & 0.570 & -0.394 & -0.348 \\
\hline NMF4 & -0.030 & 0.160 & -0.633 & 0.953 & 0.861 \\
\hline
\end{tabular}

Table 1. Correlation between NMF1, NMF3 and NMF4 scores and weights on edges.

\section{Dynamics in reduced space}

We then analyzed the dynamics in the reduced space. We divided the 3D space spanned by NMF1, 3, and 4 into $20 \times 20 \times 20$ cells, and calculated the mean velocity of the systems in each cell. The flow direction is depicted in Fig.7. In this figure, only the flows of cells that include more than 100 samples are shown. This figure shows that there is an attractive fixed point at (NMF1, NMF3, NMF4) (3.0,3.0,1.0). Interestingly, the path to this fixed point was not linear. For example, when we investigated the direction of the flow when NMF3 and 4 were small, the flow headed to the fixed point for NMF1 $\gtrsim 4$, whereas it was directed to the position $(0,0,0)$ when NMF1 $<3.0$.

To investigate these dynamics more carefully, we plotted the dynamics in the NMF1-NMF3 space in Fig.8. In this figure, the space is divided into $50 \times 50$ cells and the average velocity is calculated in each cell. First, the dynamics is very slow at (NMF1, NMF3) (0,0). The protein in this region was unfolded, and there was a large degree of freedom in motion. However, almost all motions did not contribute to folding and were ignored in the projection to the NMF1-NMF3 plane. Therefore, the velocity in the projected state becomes very slow. This result is consistent with that of our previous study. Second, there were two fixed points, (NMF1, NMF3) (2.0,0.0) and $(2.5,2.5)$. The former fixed point appeared unstable, whereas the latter was stable. However, when we investigated the 3D plot shown in Fig.7, the saddle point was not a fixed point in 3D space; When NMF4 was large, NMF1 increased at (NMF1, NMF3) (2.0,0.0), and when NMF4 was small, it decreased. By taking the average over NMF4 we obtained this "saddle". However, the latter fixed point was close to the stable fixed point in 3D space. 

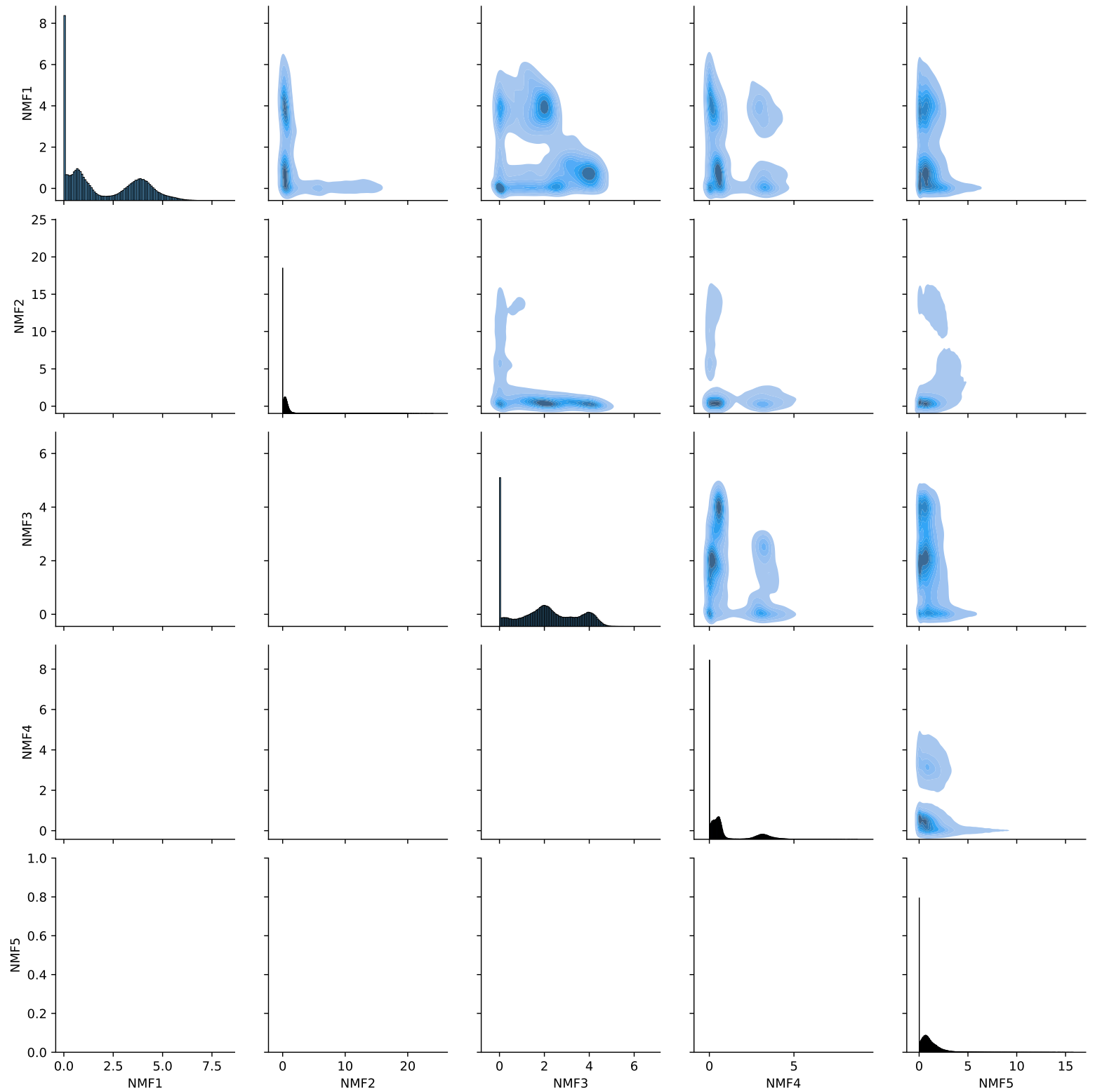

Figure 4. Density of states in reduced space by nonnegative matrix factorization (NMF). The unit of each axis was Å. Figures in diagonal part shows the histogram of each variables. 


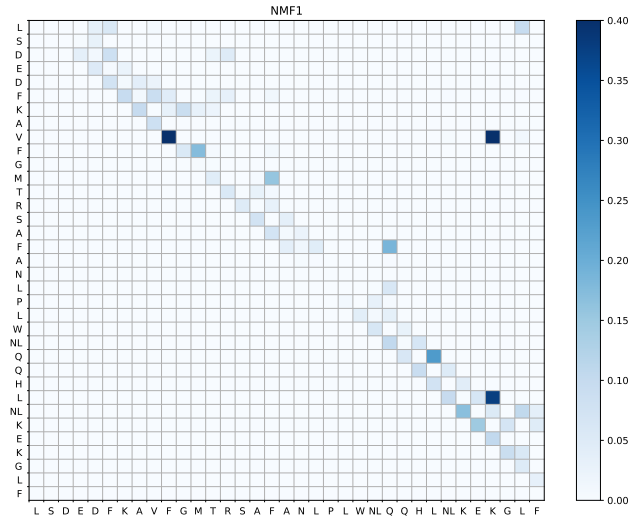

(a) NMF1

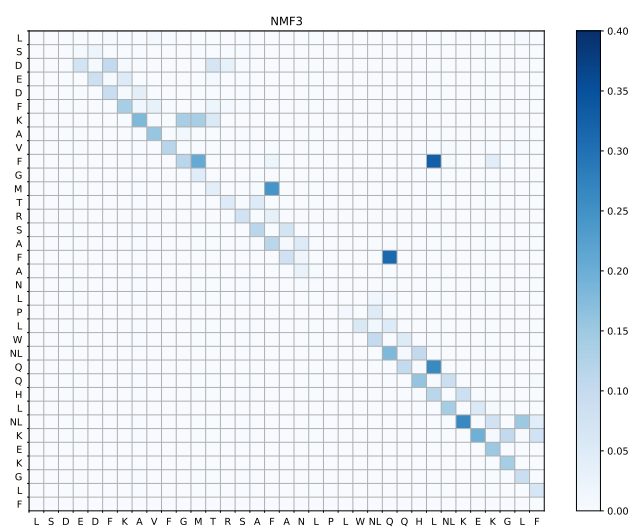

(c) NMF3

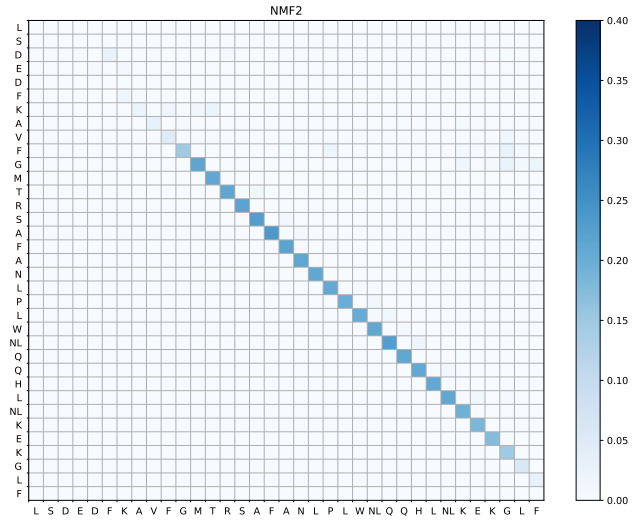

(b) NMF2

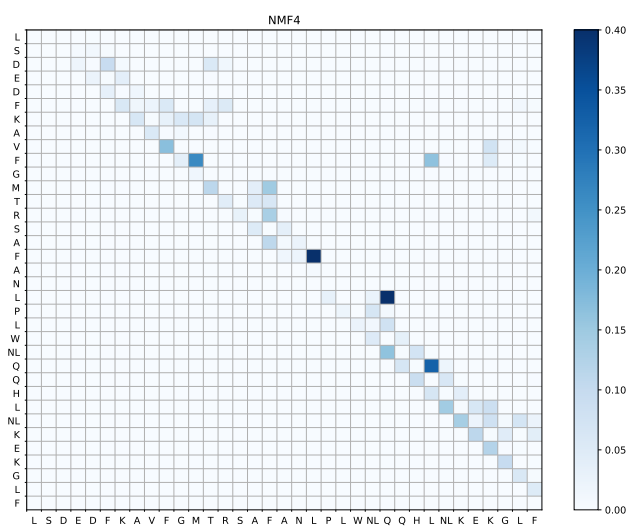

(d) NMF4

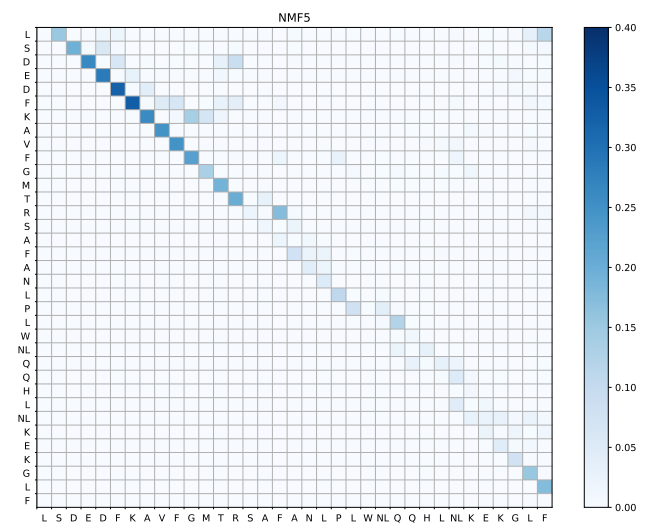

(e) NMF5

Figure 5. Basis of nonnegative matrix factorinzation(NMF). Darker cells indicate that the basis has a large component in this cell. 


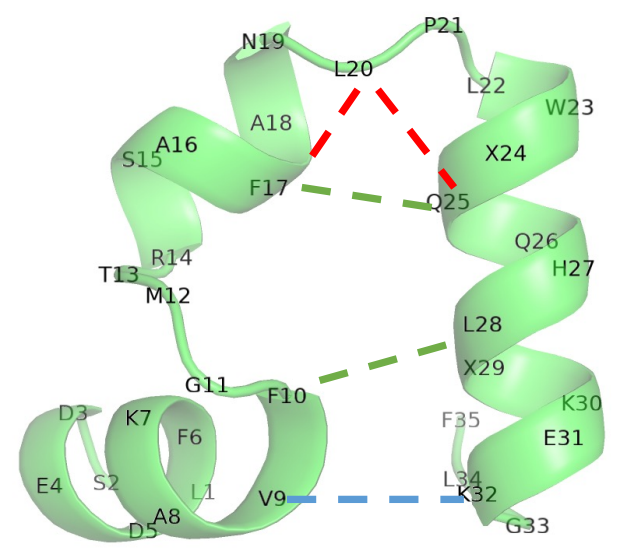

Figure 6. Native state of HP35(nle-nle). Blue, green and red dashed lines represent the pair whose component was larger than 0.3 in the 1st(blue), 3rd (green) and 4th (red) components obtained by nonnegative matrix factorization, respectively. Here, we omitted the pairs inside the $\alpha$-helices. X represents norleucine.

Third, Fig.8 shows two paths to reach the folded state. When NMF1 was larger than 2, the mean velocity headed to the fixed point, and the system approached straightly to the stable state. Because NMF1 and NMF3 scores increase when V9-L32 and F10-L28 create cycles, respectively, this result suggested that in the first path V9 and L32 made connections to form a cycle, after which F10 and L28 became close. However, when both NMF1 and NMF3 were small, the system detoured around the area $(\mathrm{NMF} 1, \mathrm{NMF} 3) \sim(2.0,1.0)$. NMF3 began to increase while the NMF1 score was kept lower than 1 in the first stage, and the NMF1 score began to increase after NMF3 21.0 . In the second stage of this path, the score of NMF3 was almost kept constant. In this path, the connection between V9 and K32 emerged after the formation of loop that included F10-L28.

\section{Discussion}

In this study, we performed the TDA of MD trajectories on the folding of the villin headpiece. Using PH and dimensional reduction by NMF, we found that the topological features of this protein coulbd be characterized by several pairs of residues. We also investigated the dynamics of this protein and found two paths for the folding process.

We carried out analyses using the point of residues, without chemical information such as hydropathy, ionization, or hydrogen bonding. Using this information, we will obtain a deeper understanding of the folding process. For example, F6, F10, and F17 form a hydrophobic core, which plays an important role in determining tertiary structure ${ }^{17}$. Among these three residues, F10 and F17 appeared to be important for determining the tertiary structure, but F6 did not. Vermeulen et al. claimed that the sequence from P21 to W23 plays an essential role in determining the structure of proteins ${ }^{18}$. The importance of the edge L20-Q25 in NMF4 appears to be consistent with this claim, but in our results, P21, L22, and W23 are not important residues. These results indicate that careful investigation is needed when combining the characteristic structure obtained through our method with chemical interactions between residues.

Our method has scope for improvement. In this method, the key idea is to treat volume optimal cycles as "text." In this approach, we describe a set of volume optimal cycles as the "text" that describes the topology embedded in the dataset. From this viewpoint, other text-mining techniques are applicable to describe the "shape" of proteins. For example, in this study the information of volume optimal cycles is vectorized by a "bag of simplices," similar to a "bag of words" in text mining. Recently, distributed embedding methods, such as Word2Vec or fasttext, have shown excellent performance in natural language processing ${ }^{19,20}$. These methods may be applicable to vectorizing PH, although slight modification is needed. Another important challenge is time series analysis combined with $\mathrm{PH}$. Applications of $\mathrm{PH}$ for time series analysis have been reported in several studies $^{21,22}$, but are currently under development. Our vectorization method enables us to apply well-established time-series 


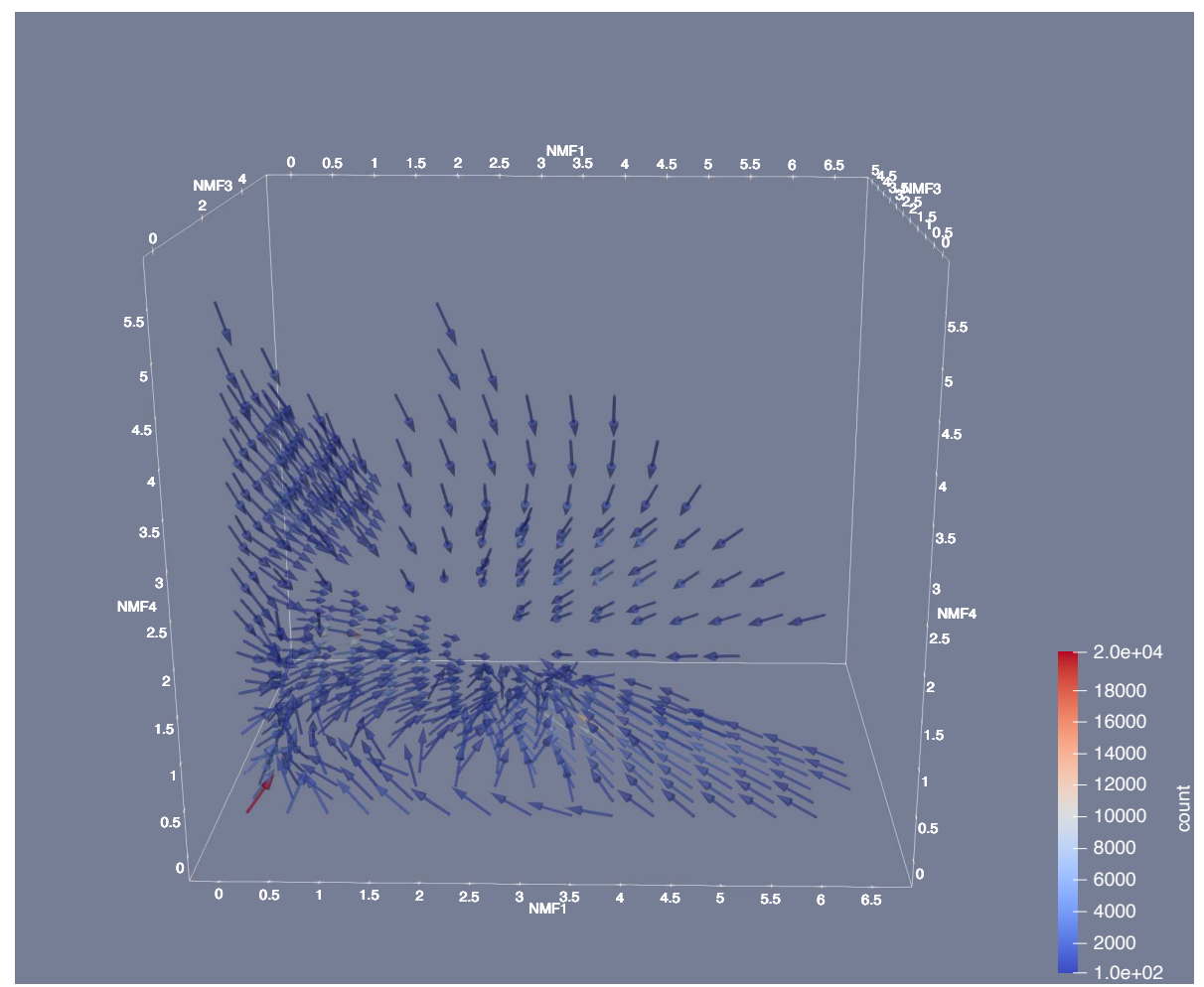

Figure 7. Average direction of flow of the systems in reduced space. The colors or arrows represent the number of samples in each cell. Only the flows of cells that include more than 100 samples are shown. The lengths of the arrows are not scaled, because the velocity at (NMF1, NMF3, NMF4) (0,0,0) was too small, thus, invisible.

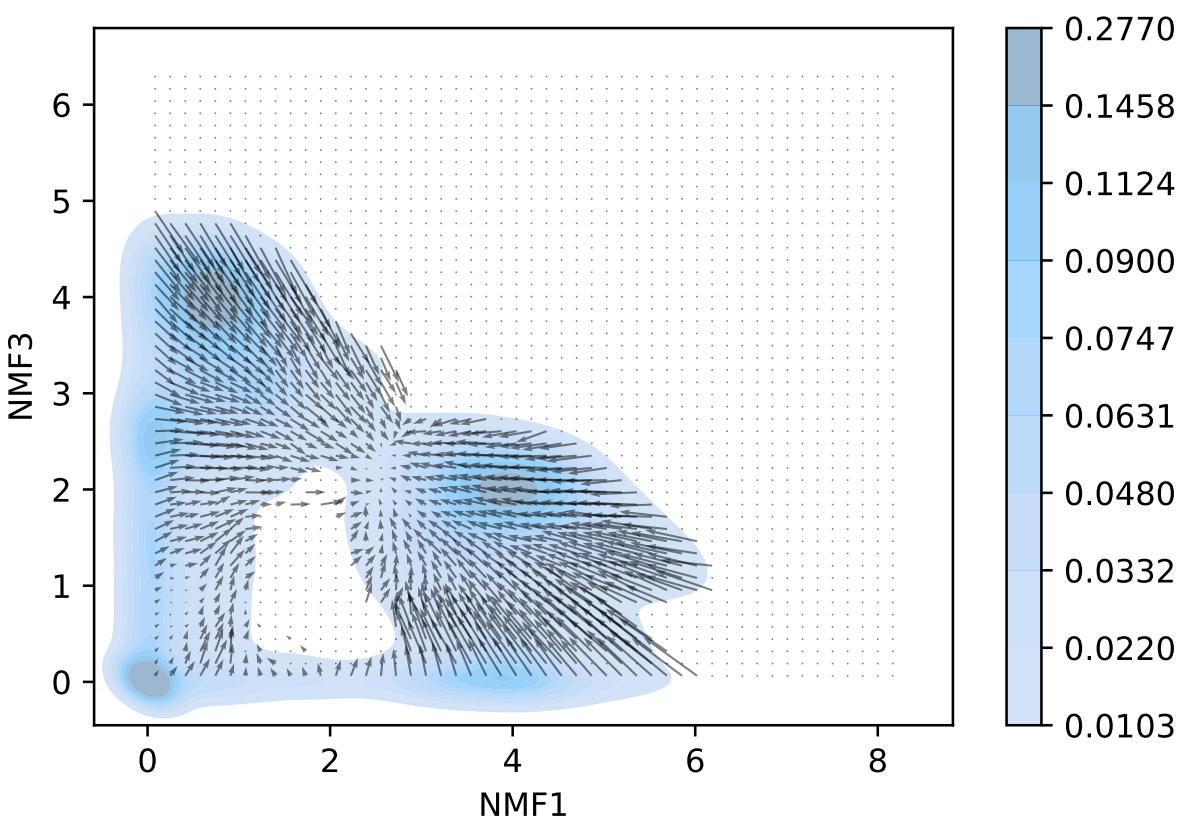

Figure 8. Average velocity in reduced space. The length of the arrow is proportional to the average velocity in the reduced space. The backgorund color indicates the density of the samples. The velocity at cells with less than 100 samples was omitted to reduce the effect of noise. 
analysis techniques, such as the state space model, which provides new insights into the dynamics of protein.

\section{References}

1. Cohen, F. E. \& Kelly, J. W. Therapeutic approaches to protein-misfolding diseases. Nature 426, 905-909, DOI: 10.1038 /nature02265 (2003).

2. Munch, E. A user's guide to topological data analysis. J. Learn. Anal. 4, 47-61 (2017).

3. Yao, Y. et al. Topological methods for exploring low-density states in biomolecular folding pathways. The J. Chem. Phys. 130, 144115, DOI: 10.1063/1.3103496 (2009).

4. Xia, K. \& Wei, G.-W. Persistent homology analysis of protein structure, flexibility and folding. Int. J. for Numer. Methods Biomed. Eng. 30, 814-844, DOI: 10.1002/cnm.2655 (2014).

5. Xia, K. \& Wei, G.-W. Multidimensional persistence in biomolecular data. J. Comput. Chem. 36, 1502-1520, DOI: $10.1002 / j c c .23953$ (2015).

6. Cang, Z. \& Wei, G.-W. TopologyNet: Topology based deep convolutional and multi-task neural networks for biomolecular property predictions. PLOS Comput. Biol. 13, e1005690, DOI: 10.1371/journal.pcbi.1005690 (2017).

7. Ichinomiya, T., Obayashi, I. \& Hiraoka, Y. Protein-Folding Analysis Using Features Obtained by Persistent Homology. Biophys. J. 118, 2926-2937, DOI: 10.1016/j.bpj.2020.04.032 (2020).

8. Beauchamp, K. A., McGibbon, R., Lin, Y.-S. \& Pande, V. S. Simple few-state models reveal hidden complexity in protein folding. Proc. Natl. Acad. Sci. 109, 17807-17813, DOI: 10.1073/PNAS.1201810109 (2012).

9. Harada, R. \& Kitao, A. The Fast-Folding Mechanism of Villin Headpiece Subdomain Studied by Multiscale Distributed Computing. J. Chem. Theory Comput. 8, 290-299, DOI: 10.1021/ct200363h (2012).

10. Wang, E., Tao, P., Wang, J. \& Xiao, Y. A novel folding pathway of the villin headpiece subdomain HP35. Phys. Chem. Chem. Phys. 21, 18219-18226 (2019).

11. Edelsbrunner, H., Letscher, D. \& Zomorodian, A. Topological Persistence and Simplification. Discret. \& Comput. Geom. 28, 511-533, DOI: 10.1007/s00454-002-2885-2 (2002).

12. Obayashi, I. Volume-Optimal Cycle: Tightest Representative Cycle of a Generator in Persistent Homology. SIAM J. on Appl. Algebr. Geom. 2, 508-534, DOI: 10.1137/17M1159439 (2018).

13. Cohen-Steiner, D., Edelsbrunner, H. \& Harer, J. Stability of Persistence Diagrams. In Proceedings of the Twenty-First Annual Symposium on Computational Geometry, SCG '05, 263-271, DOI: 10.1145/1064092.1064133 (Association for Computing Machinery, New York, NY, USA, 2005).

14. Homcloud. https://homcloud.dev/.

15. Lee, D. D. \& Seung, H. S. Learning the parts of objects by non-negative matrix factorization. Nature 401, 788 (1999).

16. Pedregosa, F. et al. Scikit-learn: Machine Learning in Python. J. Mach. Learn. Res. 12, 2825-2830 (2011).

17. Frank, B., Vardar, D., Buckley, D. \& James McKnight, C. The role of aromatic residues in the hydrophobic core of the villin headpiece subdomain. Protein Sci. 11, 680-687, DOI: 10.1110/ps.22202 (2002).

18. Vermeulen, W. et al. Identification of the PXW Sequence as a Structural Gatekeeper of the Headpiece C-terminal Subdomain Fold. J. Mol. Biol. 359, 1277-1292, DOI: https://doi.org/10.1016/j.jmb.2006.04.042 (2006).

19. Mikolov, T., Chen, K., Corrado, G. \& Dean, J. Efficient estimation of word representations in vector space. In 1st International Conference on Learning Representations, ICLR 2013 - Workshop Track Proceedings (2013).

20. Bojanowski, P., Grave, E., Joulin, A. \& Mikolov, T. Enriching Word Vectors with Subword Information. Transactions

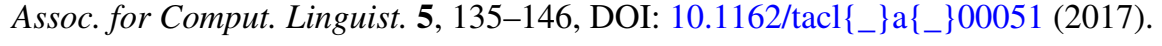

21. Perea, J. A. \& Harer, J. Sliding Windows and Persistence: An Application of Topological Methods to Signal Analysis. Foundations Comput. Math. 15, 799-838, DOI: 10.1007/s10208-014-9206-z (2015).

22. Pereira, C. M. M. \& De Mello, R. F. Persistent homology for time series and spatial data clustering. Expert. Syst. with Appl. 42, 6026-6038, DOI: 10.1016/j.eswa.2015.04.010 (2015). 


\section{Acknowledgements (not compulsory)}

The author thanks Yasuaki Hiraoka and Ippei Obayashi for their fruitful comments and advice on persistent homology, especially on the usage of Homcloud. The author also thanks to Kenji Fukumizu for his comments as a specialist in data science.

This work was financially supported by the Japan Science and Technology Agency Core Research for Evolutionary Science and Technology grant JPMJCR15D3, Japan.

\section{Author contributions statement}

T. I. conducted the planning of this study, analysis and writing.

\section{Additional information}

The author has no conflict of interest associated with this manuscript. 